\title{
The metastasis-promoting protein S100A4 regulates mammary branching morphogenesis
}

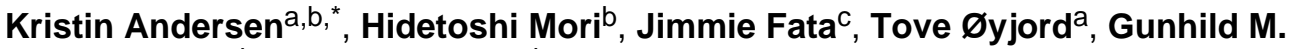 \\ Mælandsmo ${ }^{\mathrm{a}, \mathrm{d}}$, and Mina Bissell ${ }^{\mathrm{b}}$ \\ aLife Sciences Division, Lawrence Berkeley National Laboratory, Berkeley, CA, USA \\ bDepartment of Tumor Biology, Institute for Cancer Research, Oslo University Hospital, \\ Radiumhospitalet, Oslo, Norway \\ 'Department of Biology, College of Staten Island, City University of New York, New York, NY, \\ USA \\ dDepartment of Pharmacy, Faculty of Health Sciences, University of Troms $ø$, Tromsø, 9037 \\ Tromsø, Norway
}

\begin{abstract}
High levels of the S100 calcium binding protein S100A4 also called fibroblast specific protein 1 (FSP1) have been established as an inducer of metastasis and indicator of poor prognosis in breast cancer. The mechanism by which S100A4 leads to increased cancer aggressiveness has yet to be established; moreover, the function of this protein in normal mammary gland biology has not been investigated. To address the role of S100A4 in normal mammary gland, its spatial and temporal expression patterns and possible function in branching morphogenesis were investigated. We show that the protein is expressed mainly in cells of the stromal compartment of adult humans, and during active ductal development, in pregnancy and in involution of mouse mammary gland. In 3D culture models, topical addition of $\mathrm{S} 1004$ induced significant increase in the TGFa mediated branching phenotype and a concomitant increase in expression of a previously identified branching morphogen, metalloproteinase-3 (MMP-3). These events were found to be dependent on MEK activation. Downregulation of S100A4 using shRNA significantly reduced TGFa induced branching and altered E-cadherin localization. These findings provide evidence that S100A4 is developmentally regulated and that it plays a functional role in mammary gland development by, in concert with TGFa, activating MMP-3, thereby increasing invasion into the fat pad during branching. We suggest that S100A4-mediated effects during branching morphogenesis provide a plausible mechanism for how it may function in breast cancer progression.
\end{abstract}

\section{Keywords}

S100A4; Mammary gland; Branching; MMP-3; TGFa

\footnotetext{
(C) 2010 Published by Elsevier Inc.

*Corresponding author. Department of Tumor Biology, Institute for Cancer Research, Oslo University Hospital, Radiumhospitalet, Oslo, Norway. Fax: +47 22871895. kristin.andersen@ rr-research.no (K. Andersen). .

Supplementary materials related to this article can be found online at doi:10.1016/j.ydbio.2010.12.033.
} 


\section{Introduction}

S100A4, also called FSP1, is a member of the S100 family of small, calcium binding proteins. Since it was first cloned from cultured rat mammary myoepithelial cells (Barraclough et al., 1987), compelling evidence links high expression of S100A4 to increased aggressiveness of cancer cells. Experiments using transgenic animals (Ambartsumian et al., 1996; Davies et al., 1996), overexpression (Davies et al., 1993) and downregulation of S100A4 (Maelandsmo et al., 1996) have confirmed that the level of expression of this protein correlates with metastasis. In a cohort of 349 early stage breast cancer biopsies, S100A4 expression was a stronger prognostic marker than both lymph node infiltration and hormone receptor status (Rudland et al., 2000). Additionally, S100A4 was one of only 25 genes found upregulated in three different mammary cancer stem cell expression signatures (Gupta et al., 2009). Numerous other reports on clinical material from breast (Ismail et al., 2008; Lee et al., 2004), bladder (Matsumoto et al., 2007), colorectal (Flatmark et al., 2003; Gongoll et al., 2002), melanoma (Andersen et al., 2004) and several other types of cancer (Garrett et al., 2006) have confirmed the association between S100A4 expression, and a more severe prognosis. Collectively these findings suggest S100A4 as a major determinant of breast cancer progression.

The members of the $\mathrm{S} 100$ protein family undergo conformational changes during calcium binding, opening hydrophobic domains responsible for binding target proteins. Even though several binding partners have been suggested for S100A4, little is known about the molecular function of this protein. S100 proteins are expressed both intra-, and extracellularly, in a cell- and tissue-specific manner (Donato, 2003), suggesting cell- and tissue-specific roles for the different family members. S100A4 has been found in the nucleus, cytoplasm, and in the extracellular microenvironment (Cabezon et al., 2007; Flatmark et al., 2003), and in normal human tissues expression of the protein has been reported in fibroblasts (Strutz et al., 1995), endothelial cells (Ambartsumian et al., 2001), hematopoietic cells (Cabezon et al., 2007; Grigorian et al., 1994) and now in the mammary gland (this study).

Despite persistent effort from several research groups, the mechanism by which S100A4 exerts its metastasis-promoting effects has not been elucidated. Since tumors essentially utilize tissue-specific pathways and alter their regulations, we reasoned that we may be able to decipher how the protein functions by investigating its role in normal development. In this study, our goal was to investigate the expression and biological function of the protein in a normal and defined organ as a comparison to the abnormal situation during cancer onset and metastatic spread. Since S100A4 expression has the strongest impact in breast cancer, we analyzed both mice and human mammary glands for expression and regulation of S100A4 protein. In addition we used organotypic 3D cultures of primary mouse mammary epithelial cells as well as non-tumorigenic mammary epithelial cell lines from mice as functional model systems.

The mammary gland undergoes extreme changes throughout puberty, when the epithelial cells are trigged by a change in hormonal status to proliferate and invade the mesenchyme. A highly regulated process of branching morphogenesis ensues and gives rise to a tree-like structure of ducts and secondary branches. An intricate interplay between the epithelial cells and their microenvironmental factors including hormones, growth factors, cytokines, proteases and extracellular matrix (ECM) molecules, orchestrate the morphogenic process (Fata et al., 2004; Nelson et al., 2006; Simian et al., 2001; Sternlicht et al., 2006). Clearly the highly controlled branching morphogenesis and the metastatic spread of carcinoma cells have a number of similarities: both involve invasion into the surrounding tissues and the ECM, increased proliferation and involvement of metalloproteinases (MMPs). 
An exploration of the expression of S100A4 in normal mammary tissue revealed that S100A4 was expressed mainly in stromal cells of the mammary gland, and its expression peaked during the period of ductal elongation. Using three-dimensional (3D) organotypic culture models, we demonstrated that S100A4 contributes to the branching phenotype in normal epithelial cells through upregulation of MMP-3 and through modulation of Ecadherin distribution. Furthermore, we propose that tumor cells usurp the stromal role of S100A4 on branching morphogenesis and use the pathway to promote a metastatic phenotype.

\section{Materials and methods}

\section{Immunohistochemistry}

Formalin-fixed paraffin-embedded normal breast tissue were from Abcam (Cambridge, MA). Before deparaffinizing, the sections were baked for $45 \mathrm{~min}$ at $60{ }^{\circ} \mathrm{C}$. The sections were immunostained using the biotin-streptavidin-peroxidase method (Vector Laboratories, Burlin-game, CA). Deparaffinized sections were microwaved in $1 \mathrm{mM}$ EDTA (pH8.0) for $10 \mathrm{~min}$ at $800 \mathrm{~W}$ to unmask epitopes. After incubation with $1 \%$ hydrogen peroxide for 10 min, the sections were blocked with $1.5 \%$ normal horse serum in PBS for 30 min at ambient temperature, then incubated with mouse monoclonal anti-human S100A4 antibodies (22.3) (Flatmark et al., 2004), diluted 1:300, or, for the mouse sections, anti-S100A4 rabbit polyclonal antibody (Dako, Copenhagen, Denmark) diluted 1:300, in 1\% BSA in PBS at 4 ${ }^{\circ} \mathrm{C}$ overnight. The sections were thereafter incubated with biotin-labeled secondary antibody (1:200 in PBS with 1\% BSA) and strepatvidin-peroxidase (1:30) both for $30 \mathrm{~min}$ at ambient temperature. The sections were then stained with freshly prepared $0.05 \% 3,3^{\prime}$ diaminobenzidine tetrahydrocloride containing hydrogen peroxide, counterstained with hematoxylin, dehydrated and mounted. The specificity of the S100A4 antibody is described elsewhere (Flatmark et al., 2004). Negative controls were obtained by replacement of the primary antibody with mouse $\operatorname{IgG}$ of the same subclass and concentration.

\section{Brightfield microscopy}

All images were acquired using a Zeiss Axiovert200M microscope equipped with a Zeiss AxioCam color camera.

\section{Eph4 cell culture}

Eph4 cells were originally isolated from the mammary tissue of a mid-pregnant Balb/c mouse (Reichmann et al., 1989) and were a gift from E. Reichmann (Institut Suisse de Recherches, Epalinges, Switzerland). Cells were maintained in 1:1 DMEM/F12 (University of California San Francisco Cell Culture Facility), supplemented with 2\% fetal bovine serum (Invitrogen, Carlsbad, CA), $50 \mu \mathrm{g} / \mathrm{ml}$ gentamycin, and $5 \mu \mathrm{g} / \mathrm{ml}$ insulin (Sigma-Aldrich). The Eph 4 cells were used between passage 14 and 25. All shRNA transduced cell lines were used between passage 3 and 10 after infection.

\section{Isolation of primary mammary organoids}

The primary mammary organoids were isolated as described in Fata et al. (2007). In brief, the 4th inguinal mammary glands were removed from 10- to 14-week-old virgin Balb/c mice and minced (Animal protocol, \#0522 at Lawrence Berkeley Labs approved by the Animal Welfare and Research Committee (AWRC)). Minced tissue (4-8 glands) was shaken for 30 min at $37{ }^{\circ} \mathrm{C}$ in a $50-\mathrm{ml}$ collagenase/trypsin mixture ( $0.2 \%$ trypsin (Sigma, St. Louis, MO), $0.2 \%$ collagenase type IV (Gibco, Invitrogen), $5 \%$ fetal calf serum, $5 \mu \mathrm{g} / \mathrm{ml}$ insulin, $50 \mu \mathrm{g}$ / $\mathrm{ml}$ gentamycin, in $50 \mathrm{ml}$ of DMEM/F12). The collagenase solution was discarded after centrifugation at $1000 \mathrm{rpm}$, and the pellet was resuspended in $10 \mathrm{ml} \mathrm{DMEM} / \mathrm{F} 12$. The 
suspension was pelleted again at $1000 \mathrm{rpm}$ for $10 \mathrm{~min}$, resuspended in $4 \mathrm{ml}$ of DMEM/F12 + $40 \mu \mathrm{l}$ of DNase ( $2 \mathrm{U} / \mu \mathrm{l})$, and incubated for $5 \mathrm{~min}$ at ambient temperature. The DNase solution was discarded and the epithelial pieces were separated from the single cells through differential centrifugation performed at least 4 times. The final pellet was resuspended in the desired amount of medium or Matrigel (Growth Factor Reduced Matrigel®, BD Biosciences, San Jose, CA).

\section{Morphogenesis assays}

Matrigel assay-Morphogenesis assays were performed as described in Fata et al. (2007). In brief, the desired amount of primary organoids was mixed in Matrigel and added to a 96well containing an underlay of $50 \mu \mathrm{l}$ solidified Matrigel. $150 \mu \mathrm{l}$ of basal media was added to all wells (DMEM/F12 with 1\% insulin, transferrin, selenium, and 1\% penicillin/ streptomycin) for $24 \mathrm{~h}$. After $24 \mathrm{~h}$, the media was replenished (untreated samples) and growth factor, TGFa ( $9 \mathrm{nM}$; Sigma, Saint Louis, MO), and recombinant S100A4 (4 $\mu \mathrm{g} / \mathrm{ml}$ or $20 \mu \mathrm{g} / \mathrm{ml}$ for all Matrigel cultures) were added to basal media. Every other day, medium of all samples was replenished.

Collagen assay-For 3D cell cultures in $3 \mathrm{mg} / \mathrm{ml}$ collagen, primary organoids or Eph4 cell clusters were embedded in type I collagen gels as previously described (Hirai et al., 1998). In brief, the Eph 4 cells were preclustered in ultra low adhesion plates (Costar, Corning Life Sciences, Lowell, MA, USA) for $24 \mathrm{~h}$, and then collected by differential centrifugation. Acid-soluble collagen $\left(600 \mu \mathrm{l}\right.$ of a $5 \mathrm{mg} / \mathrm{ml}$ solution), Cellagen ${ }^{\mathrm{TM}}$ (AC-5, ICN, Koken, Tokyo, Japan) was mixed gently on ice with $75 \mu 1$ 10× DMEM/F12, followed by $75 \mu \mathrm{l}$ of $0.1 \mathrm{~N} \mathrm{NaOH}$. The cell clusters or organoids were suspended in DMEM/F12 at a concentration of 2500-3000 clusters per $\mathrm{ml}$, and $250 \mu \mathrm{l}$ of the suspension (or medium without clusters) was mixed with the ice-cold collagen solution. Two layers of collagen were poured into each well: a basal layer consisting only of collagen and an upper layer containing clusters of Eph4 cells. To allow solidification of the basal collagen layer, $50 \mu \mathrm{l}$ of the collagen solution was poured into each well of a 48 -well dish and incubated at $37{ }^{\circ} \mathrm{C}$ for $15 \mathrm{~min}$. Subsequently, $150 \mu \mathrm{l}$ of the $3 \mathrm{mg} / \mathrm{ml}$ cluster containing collagen suspension was poured onto the gelled basal collagen layer, and placed immediately at $37{ }^{\circ} \mathrm{C}$. After gelation, $300 \mu 1$ of chemically defined medium consisting of DMEM/F12 containing ITS (insulin/ transferrin/selenium, Sigma) with $9 \mathrm{nM}$ TGFa alone or in combination with $12 \mu \mathrm{g} / \mathrm{ml}$ recombinant S100A4 protein (rS100A4) was added. Recombinant S100A4 protein was produced and purified as previously described (Pedersen et al., 2004). As control, recombinant pro-gastrin releasing peptide (pro-GRP), a kind gift from Dr. David Warren (Central Laboratory, Oslo University Hospital, Radiumhospitalet, Oslo, Norway), or PBS, as vehicle control, was used. Medium was replenished every 2-3 days.

\section{Analysis of branching morphogenesis}

The branching phenotype of organoids embedded in Matrigel was determined after cultivation for 4-6 days, while Eph4 clusters embedded in collagen was evaluated after $72 \mathrm{~h}$. A branching phenotype was defined as an organoid or Eph4 cell cluster having at least two processes (branches) extending from its central body. Quantification of Eph4 cell branching was carried out by counting the percentage of branching clusters in each well. To determine the morphogenic response of the primary organoids, all buds on the 10 largest organoids within each well (100-200 organoids/well) were counted. All experiments were carried out in at least duplicates, and repeated at least four times. Statistical significance was determined with two tailed Student's $t$-test. All error bars represent standard error of the mean (SEM), if not stated otherwise. 


\section{Real-time reverse trancriptase PCR (qPCR)}

Total RNA was isolated with QIAGEN RNeasy Mini Kit (Quiagen, Valencia, CA). For cDNA synthesis, $10 \mathrm{ng}$ of total cellular RNA was used to synthesize cDNA using SuperScript III First-Strand Synthesis System (Invitrogen, Carlsbad, CA). S100A4 was amplified using the following forward and reverse primers

GAGCTCAAGGAGCTACTGACCA and CTCATCACCTTCTGGAATGCA; MMP3 was amplified with GTTCCTGATGTTGGTGGCTT and AGCCTTGGCTGAGTGGTAGA as forward and reverse primers, respectively. As a control for total RNA, qPCR for $18 \mathrm{~s}$ rRNA was performed with the forward and reverse primers TCGGAACTGAGGCCATGATT and CCTCCGACTTTCGTTCTTGATT. Real-time PCR was performed using LightCycler System (Roche Diagnostics, Indianapolis, IN) according to the manufacturer's instructions. Fast Start DNA Master SYBR Green I (Roche Diagnostics, Indianapolis, IN) was used in the PCR reaction. PCR data were analyzed with LightCycler Software ver.3 (Roche Diagnostics, Indianapolis, IN) and S100A4 and MMP3 expressions were quantified relative to the signals representing 18 srRNA expression.

\section{Immunocytochemical detection of E-cadherin}

Immunofluorescent analysis was performed as described in Fata et al. (2007). In brief, Eph4 cells were allowed to cluster on ultra low adhesion plates for $24 \mathrm{~h}$ as described above. Approximately 200 clusters were collected by gentle spinning, and washed once in PBS, and resuspended in $100 \mu \mathrm{l}$ PBS. $20 \mu \mathrm{l}$ of this was gently smeared on a polylysine coated glass slide. Smeared clusters were allowed to dry at ambient temperature for $15 \mathrm{~min}$ and then fixed in paraformaldehyde for $10 \mathrm{~min}$ at ambient temperature. After fixation, slides were airdried at ambient temperature for $30 \mathrm{~min}$, followed by blocking in $10 \%$ goat serum in PBS for $1 \mathrm{~h}$ at ambient temperature. The primary antibody, rat anti-E-cadherin (DECMA-1, Abcam), was incubated in blocking buffer overnight at $4^{\circ} \mathrm{C}$, followed by $3 \times 15$ min wash in $0.5 \%$ Triton- $X$ in PBS. For detection of primary antibodies, appropriate fluorophore-labeled secondary antibodies were used and confocal images were captured using a Zeiss LSM510 Meta, and Zeiss LSM and ImageJ (NIH) software.

\section{Zymography}

Culture medium conditioned by 3D Matrigel cultures for 2 days was processed for casein substrate gels as described previously (Lochter et al., 1997b; Talhouk et al., 1991) In brief, conditioned medium was mixed with zymogram sample buffer without reducing agents, incubated for $15 \mathrm{~min}$ at $37{ }^{\circ} \mathrm{C}$, and separated on $8.8 \%$ sodium dodecyl sulfate (SDS)polyacrylamide gels containing $1 \mathrm{mg} / \mathrm{ml} \beta$-casein (Sigma St. Louis, MO, USA). After electrophoresis, gels were incubated for 30 min with $2.5 \%$ Triton X-100 and subsequently for 5 days at $37^{\circ} \mathrm{C}$ in $100 \mathrm{mM}$ Tris- $\mathrm{HCl}$, pH 7.4, containing $15 \mathrm{mM} \mathrm{CaCl}_{2}$. Gels were stained with Coomassie Blue R-250 (Sigma Chemical Co., St. Louis, MO) and destained with water. Clear zones emerged against a blue background, indicating proteolytic activity.

\section{Western blotting}

Culture medium conditioned by 3D collagen cultures for 3 days was processed for immunoblotting, by mixing $15 \mu \mathrm{l}$ of conditioned medium with Western blotting sample buffer and separated on 4-20\% SDS-polyacrylamid gels (Invitrogen). Western blotting was performed as described previously (Pedersen et al., 2004). Anti-MMP-3 rabbit monoclonal antibody (Abcam, Cambridge, MA) was diluted 1:1000 in 5\% FBS in PBS, anti-S100A4 rabbit polyclonal antibody (DAKO, Copenhagen, Denmark) was diluted 1:500 in 5\% FBS in PBS, and filters incubated overnight at $4^{\circ} \mathrm{C}$. 


\section{shRNA and expression constructs}

The lentivirus for silencing S100A4 was prepared using three different Mission shRNAi constructs (Sigma-Aldrich, St. Louis, MO) with the following sequence: sh 1, CCGGCATGATGTGCAATGAATTCTTCTCGAGAAGAATTCATTGCACATCATGTTT TT; sh 2 , CCGGTGTGTCCACCTTCCACAAATACTCGAGTATTTGTGGAAGGTGGACACATT TTT; and sh3, CCGGGATGAGCAACTTGGACAGCAACTCGAGTTGCTGTCCAAGTTGCTCATCTT TTT. Stable, constitutively expressing, anti-S100A4 shRNAi-Eph4 cell lines, were generated using a ViraPower T-REx lentiviral expression system according to the manufacturer's protocol (Invitrogen Carlsbad, CA). Briefly, lentivirus with the shRNA inserts was produced by transfecting the 293FT producer cell line with the expression construct. Eph4 cells were infected with the viral supernatants, and stably transduced cells were selected using the appropriate antibiotic. To prepare expression plasmids for human S100A4, the ORF was generated using the following forward and reverse primers: ATGGCGTGCCCTCTGGAGAAG and TCATTTCTTCCTGGGCTGCT and the product was cloned via the pGem-T easy vector system (Promega, Madison, WI) and further introduced into the lentiviral expression vector pLenti CMV/TO Neo DEST \#2 puro (Invitrogen). The construct was verified by sequencing.

\section{Results}

\section{Expression of S100A4 in normal breast}

Formalin-fixed, paraffin-embedded normal human breast tissue was analyzed by immunohistochemistry, and positive S100A4 staining was detected in most, but not all fibroblasts in the breast tissue (Fig. 1A, B and C) All endothelial cells of small vessels were strongly positive (Fig. 1D). We detected strong positive staining also in big rounded cells, sometimes with granulated nuclei, presumed to be breast tissue leucocytes (Fig. 1C, D, E and F). Interestingly, we did not detect positive staining in the luminal epithelial cells of the ducts or lobules. We did not detect any staining in adipocytes. To investigate whether the expression of S100A4 in mammary gland changes during the different stages of development (ductal elongation in virgin animals, pregnancy, lactation and involution), total RNA isolated from the 4th ingual mammary gland from mice at each developmental stage was subjected to real-time reverse transcriptase PCR (qPCR). The data demonstrated the highest level of S100A4 mRNA in the mammary gland during ductal elongation (Richert et al., 2000) (Fig. 1J). Interestingly, the lowest levels of S100A4 mRNA were observed during lactation, indicating that the protein has no major biological function in the lactation process consistent with the fact that during lactation, differentiated milk producing epithelial cells of the alveoli fills most of the mammary gland and the stromal compartment is subdued (Richert et al., 2000). S100A4 mRNA levels is, however, reestablished in the involuting mammary gland, indicating a biological role for S100A4 both in the developing virgin and involuting mice.

\section{Effects of extracellular S100A4 on branching morphogenesis in mouse primary organoids}

The striking modulation of S100A4 prompted a more detailed examination of its role in the developing mammary gland. To easily manipulate the phenotype and to explore the mechanism, we utilized a 3D organotypic assay of branching morphogenesis developed in our laboratory (Fata et al., 2007). In brief, mammary gland organoids were isolated from the 4th inguinal mammary gland of 10- to 14-week-old mice and grown embedded in Matrigel. The branching process was induced after $24 \mathrm{~h}$ through addition of TGFa, a cytokine prominently expressed in the mammary gland in vivo (Snedeker et al., 1991). Addition of recombinant mouse S100A4 protein by itself did not induce branching (data not shown). 
However, when added together with TGFa, it altered the branching phenotype by increasing the number of resulting buds (Fig. 2A and B, and Supplementary Fig. 1). The presence of S100A4 in the primary cultures did not, however, change the overall percentage of branching organoids or the length of the branching tubules (results not shown). Since nonbranching organoids were unaffected, and S100A4 did not induce branching alone, we hypothesized that S100A4 stimulated a process already ongoing in the branching organoids.

Previously, MMP-3 was shown to be involved in branching morphogenesis in transgenic animals and in culture (Fata et al., 2007; Lochter et al., 1997a; Simian et al., 2001). In order to unravel factors contributing to the increased branching in the S100A4-treated cultures, qPCR was performed. S100A4 treatment in combination with TGFa induced 15-fold increase in MMP-3 expression compared to control (Fig. 2C), corresponding with an increased secretion of MMP-3 to the culture medium (Fig. 2D). Adding S100A4 alone gave only a weak induction of MMP-3, indicating a synergy between S100A4 and TGFa effects. RNA and medium from the primary organoid cultures was also tested for S100A4-mediated effects onMMP-14, -9 and -2 (results not shown). MMP-3 was the only MMP found to respond significantly to topical addition of S100A4.

\section{Effects of S100A4 on branching morphogenesis in normal mouse mammary epithelial cells}

The primary cultures of mouse organoids are highly enriched for epithelial cells. However, the cultures also contained remaining fractions of mammary gland stromal cells. In order to study the direct effect of S100A4 on mammary gland epithelial cells, and the mechanisms by which S100A4 upregulates MMP-3 and increase branching in more detail, we employed a defined assay system of normal mouse mammary epithelial cells. Preformed clusters of Eph4 cells (Reichmann et al., 1989) will, when put in 3D collagen cultures and stimulated with growth factors such as TGFa, grow and form branching duct-like structures, in a process resembling ductal branching (Nelson et al., 2006; Simian et al., 2001). When grown in $2 \mathrm{D}$ cultures on tissue culture plastic, the Eph4 cell line had endogenous expression of S100A4. In order to avoid unwanted effects of the endogenous protein, stable anti-S100A4 shRNA transduced variants (anti-S100A4 shRNAi cells, sh 1, sh 2, and sh 3), and a scrambled sh variant (sh scr) as control, were made (Supplementary Fig. 2). To further verify S100A4-specific effects, human S100A4 was reintroduced in the anti-S100A4 sh1 knockdown cell line (sh1/huA4) by a lentiviral delivered expression construct (Supplementary Fig. 2). Preformed clusters from these cell lines were put in 3D collagen gels. Interestingly, in this branching assay, using TGFa as stimulant, the control cell line transduced with the scrambled control shRNA branched very well, 57\% of the clusters had two or more branches compared to only $15 \%$ in the sh1 cell line $(p<0.045)$ (Fig. 3A and B). Furthermore, we were able to restore the branching phenotype by reintroducing S100A4 expression, or by adding recombinant S100A4 protein to the cultures (Fig. 3A and B). These results demonstrated that $\mathrm{S} 100 \mathrm{~A} 4$, in combination with TGFa, had a direct effect on the epithelial cells, increasing the branching phenotype. Furthermore, the effect was independent of the extracellular matrix composition.

Branching morphogenesis is a complicated and well-regulated process, depending on proliferation, motility and proteolysis (Fata et al., 2007; Nelson et al., 2006; Simian et al., 2001). The cell lines were therefore assayed for differences in proliferation, morphology (Supplementary Fig. 2), and motility (scratch assay, results not shown), and we detected no significant differences when grown in 2D cultures on tissue culture plastic, or in 3D without growth factor addition (results not shown). 


\section{S100A4 induced MMP-3 expression in normal mammary epithelial cell lines}

In the mouse primary organoid cultures, recombinant S100A4 induced MMP-3 expression, and probably, thereby enhanced the branching phenotype. A similar phenotypic effect of S100A4 was observed in the Eph4 cell branching assay, and to confirm the MMP-3 upregulation also in the transduced Eph4 cell lines, qPCR and immunoblotting of the conditioned cell culture medium were performed. Our results showed that both endogenously expressed (Fig. 4A), and the extracellular form of S100A4 (Fig. 4B), indeed induced MMP-3 expression in all the shRNA transduced Eph4 cells, while the human S100A4 transduced cell line (sh1/huA4), expressing high levels of S100A4 and MMP-3, did not respond to additional extracellular S100A4. Western blot of conditioned medium from the 3D collagen cultures demonstrated a corresponding increase in MMP-3 secretion (Fig. $4 \mathrm{C}$ showing the S100A4 shRNA clones sh2 and sh3 and 5D showing clone sh1). The induction of MMP-3 corresponded well to the observed increase of branching (Fig. 3B), indicating that the two events were connected.

It has previously been shown that TGFa-mediated induction of MMP-3 was dependent of signaling through mitogen-activated protein kinase (MEK) in mouse primary mammary organoids (Fata et al., 2007). Furthermore, extracellular S100A4 has been shown to induce Erk phosphorylation (Klingelhofer et al., 2009). It was therefore of interest to examine whether S100A4-mediated induction of MMP-3 was MEK-dependent, and the MEK inhibitor PD98059 was added to 3D collagen cultures of clustered sh1 cells (Fig. 5A and B).

As expected, the mRNA (not shown) and protein data (Fig. 5D, lane 1 compared to lane 3) confirmed dependency on MEK for growth factor induced MMP-3 expression. Presence of the MEK inhibitor also totally abolished the clusters ability to form branches (Fig. 5A) when stimulated with both TGFa and S100A4. Consistent with this, in the presence of MEK inhibitor, S100A4 was not able to induce MMP-3 mRNA expression (Fig. 5C). Interestingly, this result did not correspond to the protein data since extracellular MMP-3 was increased by addition of S100A4 also in the presence of PD98059 (Fig. 5D). It is therefore surprising that presence of the MEK inhibitor totally abolished the clusters ability to form branches (Fig. 5A) when stimulated with both TGFa and S100A4. It is, however, well known that inhibiting MEK also inhibits cell proliferation, and this might explain why the clusters were not able to make branches, even in the presence of extracellular MMP-3 (Fig. 5A and D, lane 2). These data indicate that MEK signaling is involved in S100A4mediated MMP-3 induction, but the discrepancy between the mRNA and protein data might also suggest that S100A4 trigger other MEK independent posttranscriptional MMP-3 regulatory pathways.

\section{S100A4 modulate cell-cell adhesion in normal mammary epithelial cell lines}

When grown as suspension cultures in low adhesion tissue culture wells for preclustering, the anti-S100A4 sh cell lines presented a phenotypic change compared to the sh scr control cell line and the Eph4 mother cell line. The control cells formed round, tight clusters with smooth edges, while sh1, sh2 and sh3 formed irregular clusters where the cell-cell contact seem to be modulated (Fig. 6A), implying that downregulation of endogenous S100A4 expression had effects on cell-cell adhesion when cells were grown in suspension. Interestingly, the sh1/huA4 cell line where S100A4 is reintroduced displayed a partially restored phenotype, forming both tight round clusters, and irregular clusters when grown in low adhesion plates. The presence of different phenotypes might be explained by variable transduction efficiency resulting in heterogeneous expression of human S100A4 in this cell line. 
S100 dimers have been suggested to act as bridging factors, bringing their binding partners together (Zimmer et al., 2003). In light of this, we tested whether addition of extracellular S100A4 to the suspension cultures where able to revert the adhesion phenotype but could not detect any effect on the intracellular adhesion in the clusters (results not shown). Seemingly, the downregulation of S100A4 gene expression did not just mechanically alter the strength of the extracellular cell-cell adhesion forces but had a more profound effect on the function of the cell-cell adhesion molecules.

The expression of S100A4 has previously been linked to loss of E-cadherin (Andersen et al., 2004; Keirsebilck et al., 1998; Kohya et al., 2003; Moriyama-Kita et al., 2005), and clusters from the different sh transduced Eph4 cell lines were therefore subjected to immunofluorecence staining with anti-E-cadherin antibodies. This revealed an alteration of E-cadherin distribution in the anti-S100A4 shRNA cell lines (Fig. 6B), where E-cadherin demonstrated a more punctuated, vesicular, pattern of distribution, which was reverted when endogenous expression of S100A4 was restored. The vesicular staining, visible in the cytoplasm from the cell membrane to the perinuclear area, indicates that removal of S100A4 interfered with the intracellular distribution of the E-cadherin molecules. Membranous Ecadherin expression was partially restored in the sh1/huA4 cell line, verifying that the change in E-cadherin distribution was caused by modulation of endogenous S100A4 expression.

\section{Discussion}

The link between expression of S100A4 in breast carcinoma cells, and poor patient outcome, has been soundly established (Ismail et al., 2008; Lee et al., 2004; Rudland et al., 2000). Since very few studies have looked at the expression and function of this protein in nonmalignant cells and tissue, the aim for the present work was to elucidate the biological function of S100A4 in normal mammary gland. The expression of S100A4 in mammary gland and during mammary gland development was investigated using immunohistochemistry and qPCR, respectively. For investigation of the biological role of S100A4 in mammary gland branching morphogenesis, we employed two model systems: a physiologically relevant, lamininrich 3D assay with primary cells (Fata et al., 2007), and Eph4 cells, a non-tumorigenic and functionally normal mouse mammary epithelial cell line, cultivated in type I collagen (Reichmann et al., 1989). In both cell systems, it was revealed that S100A4, in concert with TGFa, positively regulated mammary gland branching phenotype and caused induction of MMP-3 expression. In clusters of Eph4 cells, S100A4 caused modulation of intracellular E-cadherin distribution.

Using a highly specific anti-human S100A4 antibody (Flatmark et al., 2004), positive staining primarily in the stromal compartment of normal human breast tissue was detected. This is in concordance with previous reports using different antibodies (Cabezon et al., 2007; Pedersen et al., 2002; Rudland et al., 2000), altogether confirming the impression of S100A4 as mainly expressed in the stroma. Since the anatomy of the human and mouse mammary gland differ, we also looked at S100A4 immunostaining in normal mouse mammary gland, with similar outcome (Supplementary Fig. 3). Expression of S100A4 in early stage breast carcinoma cells has been reported as an independent marker of poor prognosis (Ismail et al., 2008; Lee et al., 2004; Rudland et al., 2000). In light of this, our results are particularly interesting, implying that during malignant transformation of mammary epithelial cells, S100A4 expression increases, giving the S100A4 expressing carcinoma cells an advantage in the metastatic process.

Mammary gland is dynamic, undergoing development and stromal rearrangements throughout life. By examining the expression of S100A4 in mouse mammary glands from 
different developmental stages, we found the highest expression during ductal elongation, whereas S100A4 expression was obliterated during lactation, meaning that the calcium binding protein is unnecessary for normal function of the terminally differentiated alveolar epithelial cells. During lactation the amount of stroma is greatly decreased (Richert et al., 2000), our finding therefore agrees with the conception of S100A4 as a stromal protein, participating in processes where the stroma undergoes rearrangements. To test this hypothesis, a physiologically relevant in vitro branching morphogenesis assay (Fata et al., 2007) was utilized, and indeed, the results demonstrated that S100A4 positively regulated the branching phenotype of primary organoids embedded in Matrigel. In light of the results discussed above, and numerous previous studies where S100A4 induces cell motility, proteolytic activity, and/or invasion in various cell types (Ambartsumian et al., 2001; Saleem et al., 2006; Schmidt-Hansen et al., 2004; Semov et al., 2005; Takenaga and Kozlova, 2006; Tarabykina et al., 2007; Zou et al., 2005), this finding may explain how S100A4 significantly contributes to the aggressiveness of mammary carcinoma cells. The obtained results show that S100A4 induces an invasive and proteolytic phenotype in normal mammary epithelial cells. During malignant transformation, the neoplastic epithelial cells respond to the increased presence of S100A4, as they are programmed to, consequently promoting their own aggressive behavior.

Primary mammary organoids are heterogenous and contain fibroblasts in addition to luminal and myoepithelial cells, making it difficult to determine the individual cell type's response to factors added into the microenvironment. To further unravel the direct extracellular and endogenous effects of S100A4 on mammary epithelial cells in a defined cellular environment, preclustered Eph4 cells undergoing TGFa induced branching morphogenesis in 3D type I collagen gels was employed. Endogenous S100A4 expression in the Eph 4 cells was minimized using anti-mouse S100A4 shRNA constructs, and a lentiviral-delivered expression vector containing human S100A4 was used to revert the knockdown phenotype and to compare the biological effects of the endogenous and extracellularly added protein. Our results demonstrated that independent of the matrix composition, the biological effect of the extracellular and the endogenously expressed protein was the same; S100A4 had a direct effect on the mammary epithelial cells, inducing MMP-3 expression, and increased the branching phenotype. Interestingly, silencing of S100A4 in the Eph4 cells reduced the branching, but not the amount of MMP-3 mRNA. It is likely that by removal of S100A4, the cells became less responsive to TGFa stimuli, while the background level of MMP-3 mRNA was not affected. When S100A4 was reintroduced, by endogenous expression, or by recombinant protein, the cells responded to the combination of TGFa and S100A4, by differentiation toward a more invasive phenotype with higher expression of proteolytic enzymes, and hence more disposed to undergo growth factor induced branching.

MMP-3 has previously been shown to induce branching morphogenesis in mouse mammary organoids, and furthermore, MMP activity was indispensable for growth factor induced mammary gland branching morphogenesis (Simian et al., 2001). It was therefore not possible to remove MMP-3 from our assay, to directly verify the link between S100A4, MMP-3 induction, and branching. We did, however, see the same association between induction of MMP-3 and S100A4 re-expression/addition of recombinant S100A4, in all antiS100A4 shRNA clones, ruling out the possibility that this effect was caused by clonal selection. Overexpression of MMP-3 in mammary gland has previously been shown to increase in vivo branching and cause increased incidence of preneoplastic lesions and invasive breast tumors compared to control mice (Sympson et al., 1995). It was later shown that MMP-3 also induced epithelial-mesenchymal transition (EMT) and malignant transformation in cultured cells (Lochter et al., 1997a; Lochter et al., 1997b; Lochter et al., 1999; Sternlicht et al., 1999). Altogether, it has been clearly demonstrated that deregulation of MMP-3 is a critical occurrence in breast tissue. This study signifies that S100A4 can act 
as an MMP-3 inducer, highlighting the significance of S100A4 deregulation in early stage breast cancer.

It was previously established that signaling through MEK was essential for MMP-3 expression in the primary organoid model system (Fata et al., 2007). By means of a MEK inhibitor, we revealed that this was the case, also in cultures of clustered Eph4 cells. When inhibiting MEK, the growth factor induced expression of MMP-3 was subdued, and the clusters did not display a branching phenotype. Interestingly, the MEK inhibitor completely blocked the S100A4 induced expression of MMP-3 mRNA, while the inhibitory effect of the protein expression was much weaker. The exact molecular mechanism by which S100A4 exert its signaling is still incompletely understood, and both NF- $\mathrm{kB}$ activation, and the MAP kinase pathways have been suggested as modulators of S100A4 signaling (Boye et al., 2008). Our data strengthen the hypothesis that MEK signaling is involved in S100A4mediated MMP-3 induction during mammary gland development, but the observed discrepancy between the mRNA and protein data might also suggest that S100A4 triggers other MEK-independent posttranscriptional pathways. Altogether, our data showed that S100A4 and TGFa had synergistic effects on expression of MMP-3 supporting the hypothesis that S100A4 is a natural participant in the cascade of events controlling the invasive process of branching morphogenesis.

When preclustering the anti-S100A4 shRNA cell lines, we observed a phenotypical change in cell-cell adhesion, combined with an alteration of E-cadherin distribution. In the sh scr control cell line, E-cadherin was concentrated to cell-cell borders, with even intensity, while the anti-S100A4 shRNA cell lines (sh1, sh2 and sh3) demonstrated a more disperse, vesicular expression in the cytoplasm, suggestive of ongoing redistribution of E-cadherin in these clusters (de Beco et al., 2009). E-cadherin and S100A4 expression have, in several cell systems, been shown inversely regulated (Keirsebilck et al., 1998; Kohya et al., 2003; Moriyama-Kita et al., 2005). In mouse mammary tumor cells, S100A4-mediated downregulation of E-cadherin favored disruption of cell-cell adhesion and subsequent stimulation of invasive capability (Keirsebilck et al., 1998).

Interestingly, S100A4 has, as MMP-3 and deregulation of E-cadherin, been associated with the onset of EMT. Transfection of murine proximal tubular epithelial cells with S100A4 gave a more elongated, fibroblast-like phenotype, and induced expression of mesenchymal markers. Moreover, cytokine-induced EMT was accompanied by de novo expression of S100A4, and when S100A4 induction was blocked, the renal epithelial cells were not able to undergo EMT (Okada et al., 1997). In light of these results, it is likely that overexpression or addition of S100A4 to mammary epithelial cells induces a more mesenchymal-like phenotype with altered cell-cell adhesion, more proteolytic activity and hence more prone to form branches.

In summary, we have shown that in normal mammary gland, S100A4 is a stromal factor contributing positively to branching morphogenesis, through induction of MMP-3 expression and E-cadherin modulation. We propose that S100A4 is one of the mediators in a tightly controlled mammary gland developmental program, driving epithelial cells towards a more invasive phenotype during branching morphogenesis. As a consequence, when this program is deregulated and exploited by the metastatic carcinoma cells, S100A4 becomes an important factor in early stage breast cancer. Our work underlines the importance of S100A4, proposes this protein as a target for anti-metastatic therapy, and calls for further elucidation of the molecular function and regulation of S100A4 in mammary gland development. 


\section{Supplementary Material}

Refer to Web version on PubMed Central for supplementary material.

\section{Acknowledgments}

The authors wish to thank Dr. Jamie Bascomb for providing the RNA from CD1 mice mammary gland and Jamie Inman for providing mouse mammary gland tissue sections. The work from MJB's laboratory is supported by grants from the U.S. Department of Energy, Office of Biological and Environmental Research (DEAC02-05CH1123), a Distinguished Fellow Award and Low Dose Radiation Program (03-76SF00098); by National Cancer Institute awards R01CA064786, R01CA057621, U54CA126552, U54CA112970 and U54CA143836 and by U.S. Department of Defense (W81XWH0810736).The work was furthermore supported by the Norwegian Cancer Society (grant no A-05121 to KA and PR-2006-0272 to GMM), the National Program for Research on Functional Genomics (\#158954/S10) and the Program for Centers for Research-based Innovation (\#174938) of the Norwegian Research Council (to G.M.M.), and the Jeanette and Søren Bothners Legacy (to G.M.M.).

\section{References}

Ambartsumian NS, Grigorian MS, Larsen IF, Karlstrom O, Sidenius N, Rygaard J, Georgiev G, Lukanidin E. Metastasis of mammary carcinomas in GRS/A hybrid mice transgenic for the mts 1 gene. Oncogene. 1996; 13:1621-1630. [PubMed: 8895507]

Ambartsumian N, Klingelhofer J, Grigorian M, Christensen C, Kriajevska M, Tulchinsky E, Georgiev G, Berezin V, Bock E, Rygaard J, Cao R, Cao Y, Lukanidin E. The metastasis-associated Mts1(S100A4) protein could act as an angiogenic factor. Oncogene. 2001; 20:4685-4695. [PubMed: 11498791]

Andersen K, Nesland JM, Holm R, Florenes VA, Fodstad O, Maelandsmo GM. Expression of S100A4 combined with reduced E-cadherin expression predicts patient outcome in malignant melanoma. Mod. Pathol. 2004; 17:990-997. [PubMed: 15133476]

Barraclough R, Savin J, Dube SK, Rudland PS. Molecular cloning and sequence of the gene for p9Ka. A cultured myoepithelial cell protein with strong homology to S-100, a calcium-binding protein. J. Mol. Biol. 1987; 198:13-20. [PubMed: 3430604]

Boye K, Grotterod I, Aasheim HC, Hovig E, Maelandsmo GM. Activation of NF-kappaB by extracellular S100A4: analysis of signal transduction mechanisms and identification of target genes. Int. J. Cancer. 2008; 123:1301-1310. [PubMed: 18548584]

Cabezon T, Celis JE, Skibshoj I, Klingelhofer J, Grigorian M, Gromov P, Rank F, Myklebust JH, Maelandsmo GM, Lukanidin E, Ambartsumian N. Expression of S100A4 by a variety of cell types present in the tumor microenvironment of human breast cancer. Int. J. Cancer. 2007; 121:14331444. [PubMed: 17565747]

Davies BR, Barraclough R, Davies MP, Rudland PS. Production of the metastatic phenotype by DNA transfection in a rat mammary model. Cell Biol. Int. 1993; 17:871-879. [PubMed: 8220314]

Davies MP, Rudland PS, Robertson L, Parry EW, Jolicoeur P, Barraclough R. Expression of the calcium-binding protein S100A4 (p9Ka) in MMTV-neu transgenic mice induces metastasis of mammary tumours. Oncogene. 1996; 13:1631-1637. [PubMed: 8895508]

de Beco S, Gueudry C, Amblard F, Coscoy S. Endocytosis is required for E-cadherin redistribution at mature adherens junctions. Proc. Natl Acad. Sci. USA. 2009; 106:7010-7015. [PubMed: 19372377]

Donato R. Intracellular and extracellular roles of S100 proteins. Microsc. Res. Tech. 2003; 60:540551. [PubMed: 12645002]

Fata JE, Werb Z, Bissell MJ. Regulation of mammary gland branching morphogenesis by the extracellular matrix and its remodeling enzymes. Breast Cancer Res. 2004; 6:1-11. [PubMed: 14680479]

Fata JE, Mori H, Ewald AJ, Zhang H, Yao E, Werb Z, Bissell MJ. The MAPK (ERK-1, 2) pathway integrates distinct and antagonistic signals from TGFalpha and FGF7 in morphogenesis of mouse mammary epithelium. Dev. Biol. 2007; 306:193-207. [PubMed: 17448457]

Flatmark K, Pedersen KB, Nesland JM, Rasmussen H, Aamodt G, Mikalsen SO, Bjornland K, Fodstad O, Maelandsmo GM. Nuclear localization of the metastasis-related protein S100A4 correlates with tumour stage in colorectal cancer. J. Pathol. 2003; 200:589-595. [PubMed: 12898594] 
Flatmark K, Maelandsmo GM, Mikalsen SO, Nustad K, Varaas T, Rasmussen H, Meling GI, Fodstad O, Paus E. Immunofluorometric assay for the metastasis-related protein S100A4: release of S100A4 from normal blood cells prohibits the use of S100A4 as a tumor marker in plasma and serum. Tumour Biol. 2004; 25:31-40. [PubMed: 15192310]

Garrett SC, Varney KM, Weber DJ, Bresnick AR. S100A4, a mediator of metastasis. J. Biol. Chem. 2006; 281:677-680. [PubMed: 16243835]

Gongoll S, Peters G, Mengel M, Piso P, Klempnauer J, Kreipe H, von Wasielewski R. Prognostic significance of calcium-binding protein S100A4 in colorectal cancer. Gastroenterology. 2002; 123:1478-1484. [PubMed: 12404222]

Grigorian M, Tulchinsky E, Burrone O, Tarabykina S, Georgiev G, Lukanidin E. Modulation of mts1 expression in mouse and human normal and tumor cells. Electrophoresis. 1994; 15:463-468. [PubMed: 8055872]

Gupta PB, Onder TT, Jiang G, Tao K, Kuperwasser C, Weinberg RA, Lander ES. Identification of selective inhibitors of cancer stem cells by high-throughput screening. Cell. 2009; 138:645-659. [PubMed: 19682730]

Hirai Y, Lochter A, Galosy S, Koshida S, Niwa S, Bissell MJ. Epimorphin functions as a key morphoregulator for mammary epithelial cells. J. Cell Biol. 1998; 140:159-169. [PubMed: 9425164]

Ismail NI, Kaur G, Hashim H, Hassan MS. S100A4 overexpression proves to be independent marker for breast cancer progression. Cancer cell international. 2008; 8:12. [PubMed: 18771601]

Keirsebilck A, Bonne S, Bruyneel E, Vermassen P, Lukanidin E, Mareel M, van Roy F. E-cadherin and metastasin (mts-1/S100A4) expression levels are inversely regulated in two tumor cell families. Cancer Res. 1998; 58:4587-4591. [PubMed: 9788607]

Klingelhofer J, Moller HD, Sumer EU, Berg CH, Poulsen M, Kiryushko D, Soroka V, Ambartsumian N, Grigorian M, Lukanidin EM. Epidermal growth factor receptor ligands as new extracellular targets for the metastasis-promoting S100A4 protein. FEBS J. 2009; 276:5936-5948. [PubMed: 19740107]

Kohya N, Kitajima Y, Jiao W, Miyazaki K. Effects of E-cadherin transfection on gene expression of a gallbladder carcinoma cell line: repression of MTS1/S100A4 gene expression. Int. J. Cancer. 2003; 104:44-53. [PubMed: 12532418]

Lee WY, Su WC, Lin PW, Guo HR, Chang TW, Chen HH. Expression of S100A4 and Met: potential predictors for metastasis and survival in early-stage breast cancer. Oncology. 2004; 66:429-438. [PubMed: 15452371]

Lochter A, Galosy S, Muschler J, Freedman N, Werb Z, Bissell MJ. Matrix metalloproteinase stromelysin-1 triggers a cascade of molecular alterations that leads to stable epithelial-tomesenchymal conversion and a premalignant phenotype in mammary epithelial cells. J. Cell Biol. 1997a; 139:1861-1872. [PubMed: 9412478]

Lochter A, Srebrow A, Sympson CJ, Terracio N, Werb Z, Bissell MJ. Misregulation of stromelysin-1 expression in mouse mammary tumor cells accompanies acquisition of stromelysin-1-dependent invasive properties. J. Biol. Chem. 1997b; 272:5007-5015. [PubMed: 9030563]

Lochter A, Werb Z, Bissell MJ. Transcriptional regulation of stromelysin-1 gene expression is altered during progression of mouse mammary epithelial cells from functionally normal to malignant. Matrix Biol. 1999; 18:455-467. [PubMed: 10601733]

Maelandsmo GM, Hovig E, Skrede M, Engebraaten O, Florenes VA, Myklebost O, Grigorian M, Lukanidin E, Scanlon KJ, Fodstad O. Reversal of the in vivo metastatic phenotype of human tumor cells by an anti-CAPL (mts1) ribozyme. Cancer Res. 1996; 56:5490-5498. [PubMed: 8968106]

Matsumoto K, Irie A, Satoh T, Ishii J, Iwabuchi K, Iwamura M, Egawa S, Baba S. Expression of S100A2 and S100A4 predicts for disease progression and patient survival in bladder cancer. Urology. 2007; 70:602-607. [PubMed: 17688917]

Moriyama-Kita M, Endo Y, Yonemura Y, Heizmann CW, Miyamori H, Sato H, Yamamoto E, Sasaki T. S100A4 regulates E-cadherin expression in oral squamous cell carcinoma. Cancer Lett. 2005; 230:211-218. [PubMed: 16297707] 
Nelson CM, Vanduijn MM, Inman JL, Fletcher DA, Bissell MJ. Tissue geometry determines sites of mammary branching morphogenesis in organotypic cultures. Sci. NY N. Y. 2006; 314:298-300.

Okada H, Danoff TM, Kalluri R, Neilson EG. Early role of Fsp1 in epithelial-mesenchymal transformation. Am. J. Physiol. 1997; 273:F563-F574. [PubMed: 9362334]

Pedersen KB, Nesland JM, Fodstad O, Maelandsmo GM. Expression of S100A4, E-cadherin, alphaand beta-catenin in breast cancer biopsies. Br. J. Cancer. 2002; 87:1281-1286. [PubMed: 12439718]

Pedersen KB, Andersen K, Fodstad O, Maelandsmo GM. Sensitization of interferon-gamma induced apoptosis in human osteosarcoma cells by extracellular S100A4. BMC Cancer. 2004; 4:52. [PubMed: 15318945]

Reichmann E, Ball R, Groner B, Friis RR. New mammary epithelial and fibroblastic cell clones in coculture form structures competent to differentiate functionally. J. Cell Biol. 1989; 108:11271138. [PubMed: 2466037]

Richert MM, Schwertfeger KL, Ryder JW, Anderson SM. An atlas of mouse mammary gland development. J. Mammary Gland Biol. Neoplasia. 2000; 5:227-241. [PubMed: 11149575]

Rudland PS, Platt-Higgins A, Renshaw C, West CR, Winstanley JH, Robertson L, Barraclough R. Prognostic significance of the metastasis-inducing protein S100A4 (p9Ka) in human breast cancer. Cancer Res. 2000; 60:1595-1603. [PubMed: 10749128]

Saleem M, Kweon MH, Johnson JJ, Adhami VM, Elcheva I, Khan N, Bin Hafeez B, Bhat KM, Sarfaraz S, Reagan-Shaw S, Spiegelman VS, Setaluri V, Mukhtar H. S100A4 accelerates tumorigenesis and invasion of human prostate cancer through the transcriptional regulation of matrix metalloproteinase 9. Proc. Natl Acad. Sci. USA. 2006; 103:14825-14830. [PubMed: 16990429]

Schmidt-Hansen B, Ornas D, Grigorian M, Klingelhofer J, Tulchinsky E, Lukanidin E, Ambartsumian N. Extracellular S100A4(mts1) stimulates invasive growth of mouse endothelial cells and modulates MMP-13 matrix metalloproteinase activity. Oncogene. 2004; 23:5487-5495. [PubMed: 15122322]

Semov A, Moreno MJ, Onichtchenko A, Abulrob A, Ball M, Ekiel I, Pietrzynski G, Stanimirovic D, Alakhov V. Metastasis-associated protein S100A4 induces angiogenesis through interaction with Annexin II and accelerated plasmin formation. J. Biol. Chem. 2005; 280:20833-20841. [PubMed: 15788416]

Simian M, Hirai Y, Navre M, Werb Z, Lochter A, Bissell MJ. The interplay of matrix metalloproteinases, morphogens and growth factors is necessary for branching of mammary epithelial cells. Dev. Camb. Engl. 2001; 128:3117-3131.

Snedeker SM, Brown CF, DiAugustine RP. Expression and functional properties of transforming growth factor alpha and epidermal growth factor during mouse mammary gland ductal morphogenesis. Proc. Natl Acad. Sci. USA. 1991; 88:276-280. [PubMed: 1986376]

Sternlicht MD, Lochter A, Sympson CJ, Huey B, Rougier JP, Gray JW, Pinkel D, Bissell MJ, Werb Z. The stromal proteinase MMP3/stromelysin-1 promotes mammary carcinogenesis. Cell. 1999; 98:137-146. [PubMed: 10428026]

Sternlicht MD, Kouros-Mehr H, Lu P, Werb Z. Hormonal and local control of mammary branching morphogenesis. Differ. research biological diversity. 2006; 74:365-381.

Strutz F, Okada H, Lo CW, Danoff T, Carone RL, Tomaszewski JE, Neilson EG. Identification and characterization of a fibroblast marker: FSP1. J. Cell Biol. 1995; 130:393-405. [PubMed: 7615639]

Sympson CJ, Bissell MJ, Werb Z. Mammary gland tumor formation in transgenic mice overexpressing stromelysin-1. Semin. Cancer Biol. 1995; 6:159-163. [PubMed: 7495984]

Takenaga K, Kozlova EN. Role of intracellular S100A4 for migration of rat astrocytes. Glia. 2006; 53:313-321. [PubMed: 16265672]

Talhouk RS, Chin JR, Unemori EN, Werb Z, Bissell MJ. Proteinases of the mammary gland: developmental regulation in vivo and vectorial secretion in culture. Dev. Camb. Engl. 1991; 112:439-449. 
Tarabykina S, Griffiths TR, Tulchinsky E, Mellon JK, Bronstein IB, Kriajevska M. Metastasisassociated protein S100A4: spotlight on its role in cell migration. Curr. Cancer Drug Targets. 2007; 7:217-228. [PubMed: 17504119]

Zimmer DB, Wright Sadosky P, Weber DJ. Molecular mechanisms of S100-target protein interactions. Microsc. Res. Tech. 2003; 60:552-559. [PubMed: 12645003]

Zou M, Al-Baradie RS, Al-Hindi H, Farid NR, Shi Y. S100A4 (Mts1) gene overexpression is associated with invasion and metastasis of papillary thyroid carcinoma. Br. J. Cancer. 2005; 93:1277-1284. [PubMed: 16265347] 

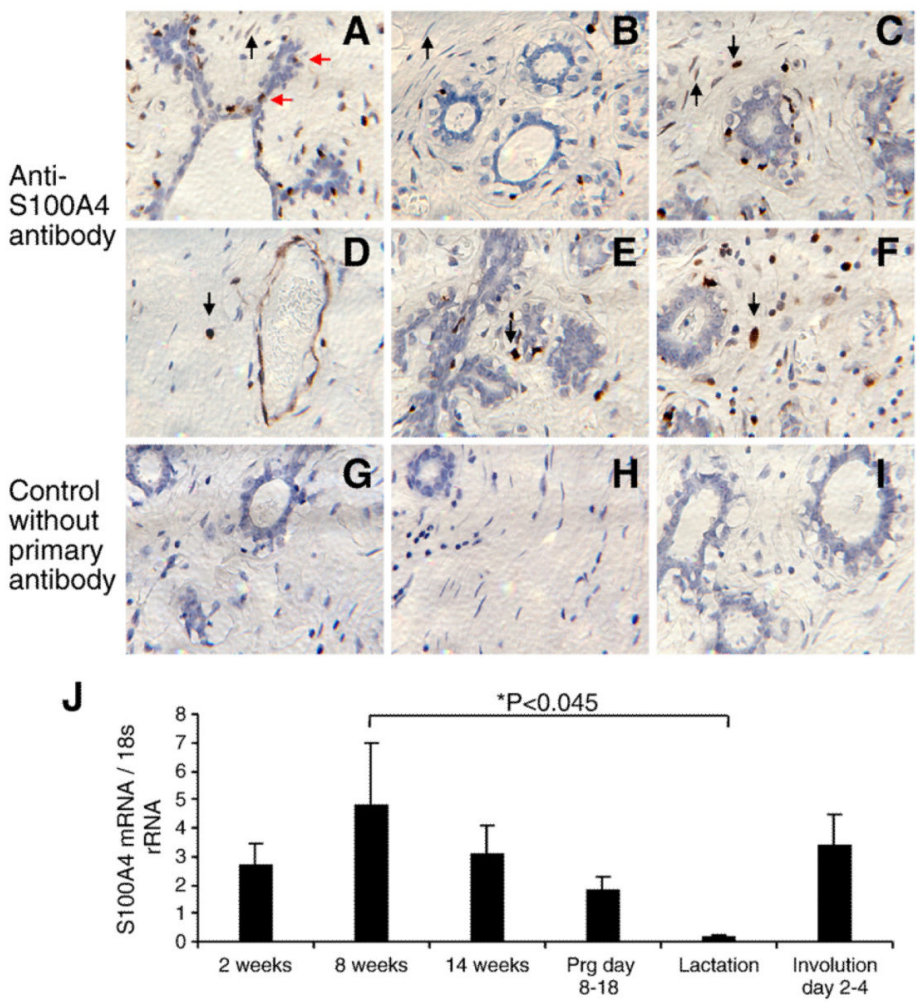

Fig. 1.

Expression of S100A4 in normal mammary gland. Representative brightfield images of formalin-fixed paraffin-embedded normal human breast tissue sections (Abcam) analyzed by immunohistochemistry for expression of S100A4 protein, using an in-house monoclonal antibody (22.3) (Flatmark et al., 2004). (A) Positive staining in occasional cells adjacent to luminal epithelial cells, possibly tissue leucocytes (red arrows). (A, B and C) Positive staining was detected in most but not all fibroblasts in the breast tissue, (black arrows pointing upwards, A and C positive staining, B negative staining). (D) All the endothelial cells of small vessels were strongly positive. (C, D, E, F) Throughout the breast tissue, we detected strong positive staining in big, rounded cells, sometimes granulated, assumingly breast tissue leucocytes (black arrows pointing down). (G-I) Control without primary antibody. (J) Expression of S100A4 in normal mouse mammary gland development. qPCR using total RNA isolated from the 4th ingual mammary gland from CD1 mice of each developmental stage and mouse S100A4 primers. The amount of S00A4 mRNA was normalized to $18 \mathrm{~s}$ RNA. Error bars are SEM. Mammary glands from at least two animals in each developmental stage. 

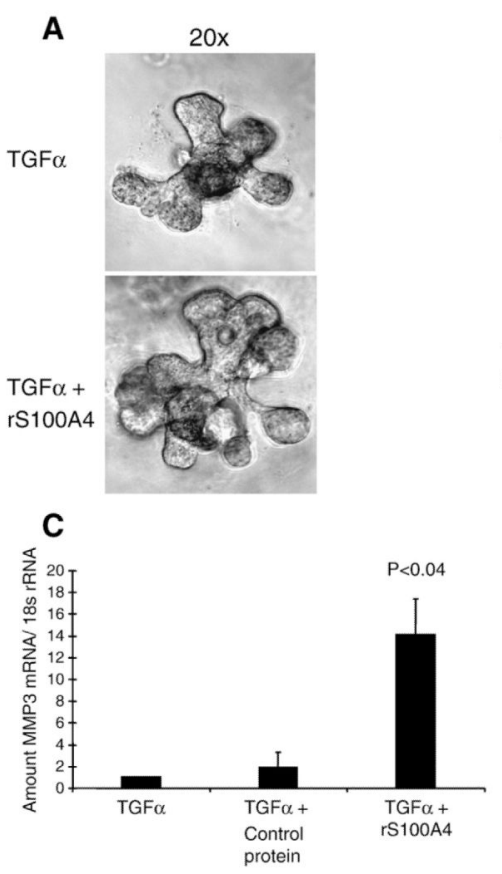

B

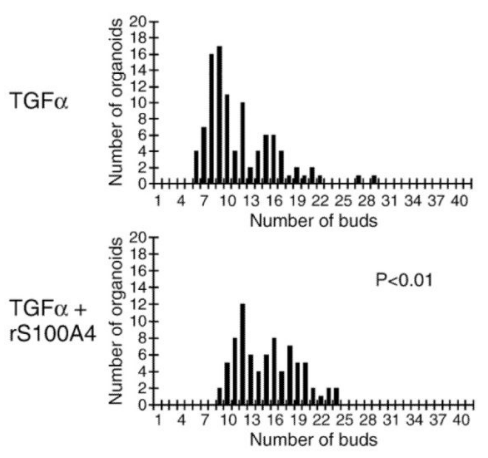

D

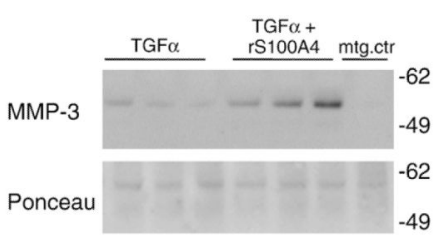

Fig. 2.

Effects of extracellular S100A4 on branching morphogenesis in primary mouse mammary gland organoids grown in 3D matrigel cultures. (A) Brightfield images of representative organoids, $96 \mathrm{~h}$ after addition of $4 \mu \mathrm{g} / \mathrm{ml} \mathrm{S100A} 4$ in combination with TGFa, to the culture medium, compared to TGFa alone. (B) Quantification of branching morphogenesis after treatment of the organoids with S100A4 protein in combination with TGFa. The frequency plots illustrate number of buds on the ten largest organoids in each well. Mean number of buds was significantly higher in S100A4 treated compared to control wells (15.1 vs. 11.6) $(p<0.01)$. (C) S100A4 induced MMP-3 mRNA expression from primary mammary gland organoids. qPCR with mouse MMP-3 primers on total RNA from primary mammary gland organoid cultures treated with $4 \mu \mathrm{g} / \mathrm{ml}$ recombinant S100A4 protein for $96 \mathrm{~h}$, compared to pro-GRP as control protein (a protein of same size, with the same his tag, and produced in the same manner as the recombinant S100A4 protein) and vehicle control (PBS). (D) A representative Western gel of medium from primary organoid cultures, stained with anti MMP-3 antibody. The ponceau staining is shown as a loading control. $15 \mu 1$ from three parallel wells from one experiment was loaded on the gel. 

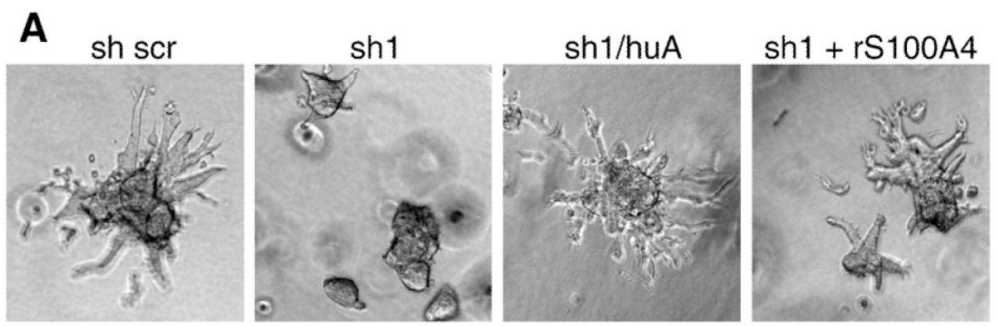

B

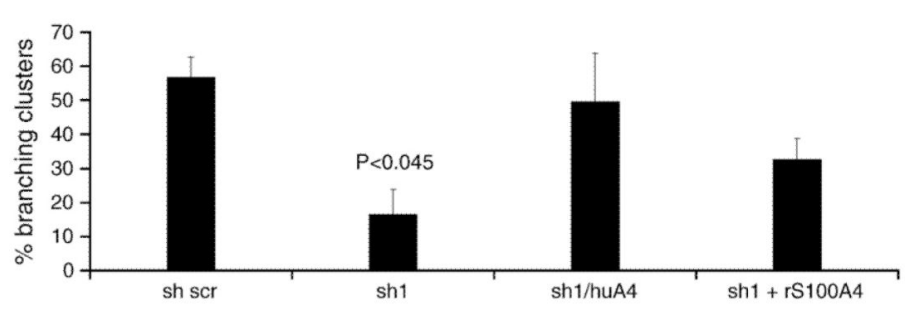

Fig. 3.

S100A4 induces branching morphogenesis of clustered mammary epithelial cells grown in 3D collagen cultures. Preclustered Eph4 clones were seeded into $3 \mathrm{mg} / \mathrm{ml}$ collagen gels and allowed to branch. (A) Representative images of clusters from shRNA transduced Eph4 cells cultured in $3 \mathrm{mg} / \mathrm{ml}$ collagen for $72 \mathrm{~h}$. (B). Quantification of the percentage of branching clusters in the 3D collagen cultures in A. All clusters in a well having more than two branches were counted and downregulation of S100A4 was found to significantly reduce the Eph4 cells ability to undergo branching morphogenesis $(P<0.045)$. 

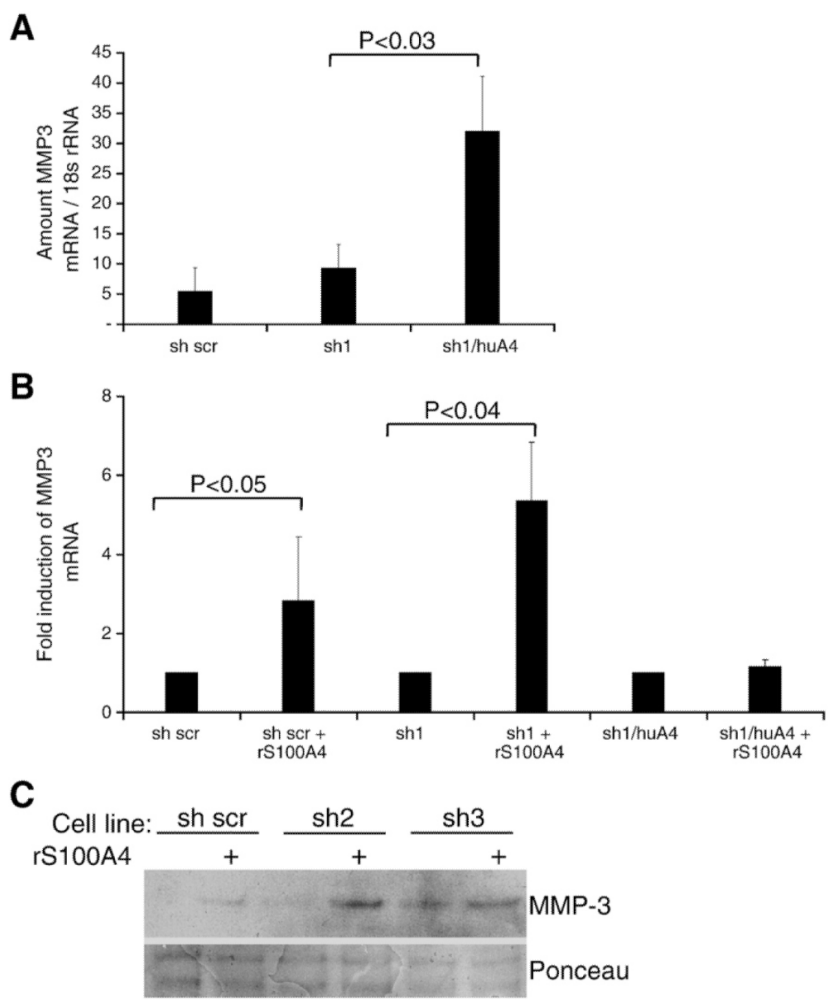

Fig. 4.

S100A4 induces MMP-3 expression in mammary epithelial cells grown in 3D collagen cultures. (A) Effect of endogenously expressed S100A4 and (B) effect of extracellular $\mathrm{S} 100 \mathrm{~A} 4(12 \mu \mathrm{g} / \mathrm{ml})$ on MMP-3 expression in 3D cultures treated with TGFa, as measured by qPCR. The sh1/huA4 cell line expressed significantly higher amounts of MMP3 mRNA compared to sh scr and sh1 cells, while the sh scr and sh1 cells responded to topical addition of recombinant S100A4 by a significant increase in MMP3 mRNA. C) Western blot of conditioned medium from 3D collagen cultures of control and two different shRNA transduced cell lines treated with or without $12 \mu \mathrm{g} / \mathrm{ml} \mathrm{recombinant} \mathrm{S100A4}$ in combination with TGFa. The immunoblot was labeled with anti MMP-3 antibody. Ponceau staining was used as loading control. 


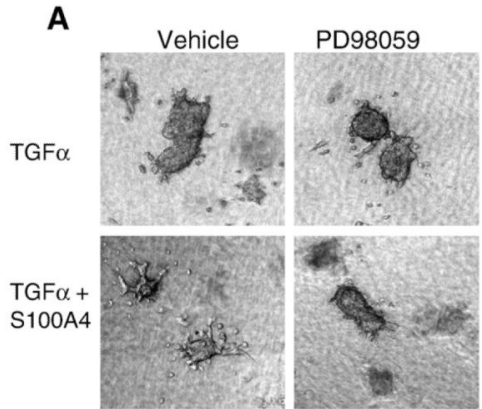

C

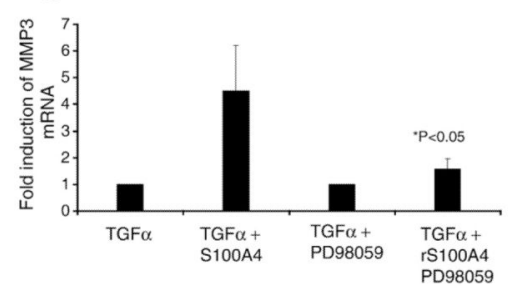

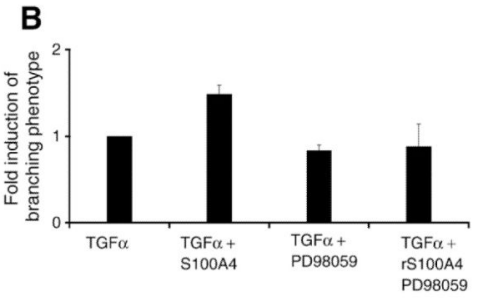

D

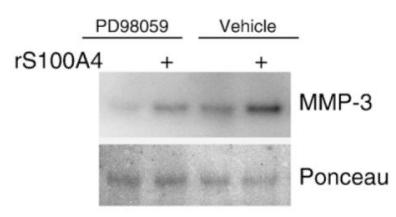

Fig. 5.

S100A4 induced branching was dependent of MEK activity. (A) Brightfield images of

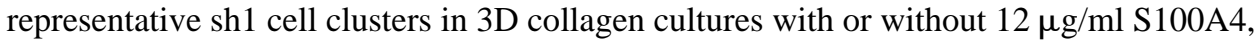
and/or the $25 \mu \mathrm{M}$ of the MEK inhibitor PD98059 (Cell Signaling Technology, Inc. Danvers, MA) in combination with TGFa. Magnification is 10×. (B) Quantification of the percentage of branching clusters in 3D collagen cultures of sh1 cells treated as indicated in graph. (C) qPCR using MMP3 primers on total RNA isolated from cultures in B. The induction of MMP3 mRNA was significantly lower in the presence of the MEK inhibitor, (1.4-fold vs. 4.3-fold in the control, $P<0.05$ ). (D) Representative Western blot of conditioned medium from cultures in B, labeled with anti MMP-3 antibody. Ponceau was used as loading control. 

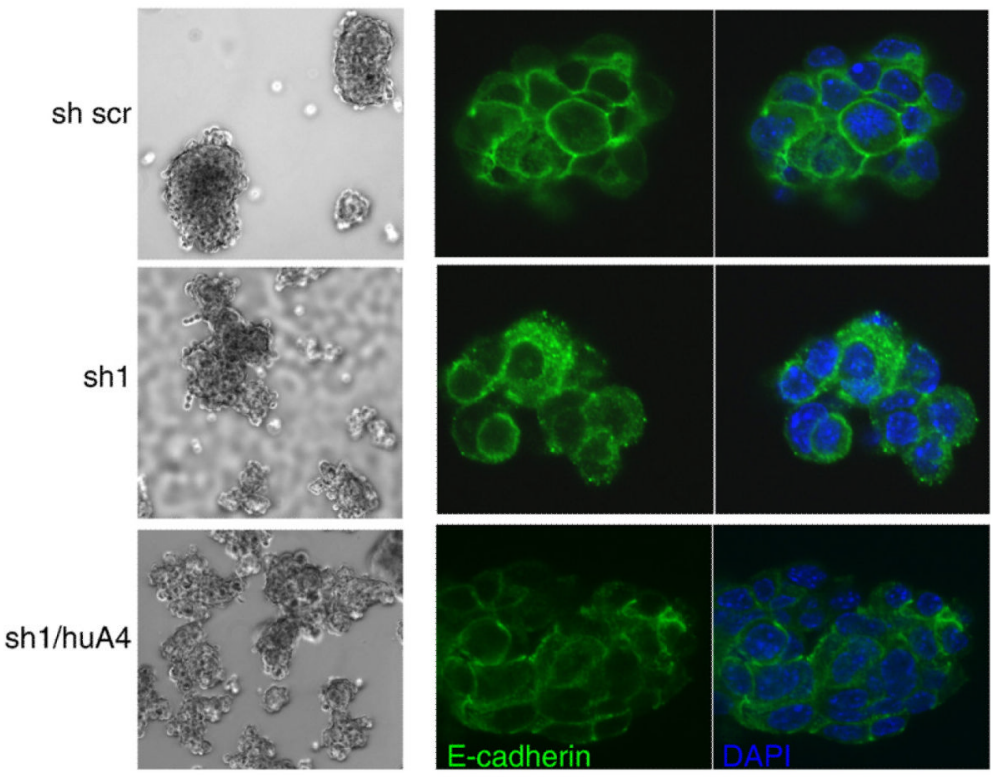

Fig. 6.

S100A4 affects cell-cell adhesion molecules. Left side: brightfield images of clustered mammary epithelial cells (Eph4 cells), in suspension cultures. The Eph4 cells were transduced with anti-S100A4 shRNA (sh1) and scrambeld shRNA (sh scr) as control. The bottom panel is sh1 cells overexpressing human S100A4 (sh1/huA4). Right side: confocal images of clusters stained with an anti-E-cadherin antibody (green), visualizing intracellular distribution of E-cadherin. The nuclei are counterstained with DAPI (blue). 